OPEN ACCESS

$$
\begin{array}{r}
\text { Edited by: } \\
\text { Zuhong He, } \\
\text { Reviewed by: } \\
\text { Elaine Y. M. Wong, } \\
\text { Australia } \\
\text { Tihua Zheng, } \\
\text { Ear Science Institute Australia, } \\
\text { Huizhan Liu, } \\
\text { Binzhou Medical University, China } \\
\text { Creighton University, United States } \\
\text { Le Xie, } \\
\text { Huazhong University of Science } \\
\text { and Technology, China } \\
\text { *Correspondence: } \\
\text { Renjie Chai } \\
\text { renjiec@seu.EdU.cn } \\
\text { Shasha Zhang } \\
\text { zhangss5576@163.com } \\
\text { Ling Lu } \\
\text { entluling60@126.com } \\
\text { He Li }
\end{array}
$$

Received: 03 July 2021 Accepted: 14 September 2021 Published: 01 October 2021

Citation: Chen Y, Qiang R, Zhang Y, Cao W, Wu L, Jiang P, Ai J, Ma X, Dong Y,

Gao X, Li H, Lu L, Zhang S and

Chai $R$ (2021) The Expression and Roles of the Super Elongation Complex in Mouse Cochlear Lgr5+

Progenitor Cells.

Front. Cell. Neurosci. 15:735723. doi: 10.3389/fncel.2021.735723

\section{The Expression and Roles of the Super Elongation Complex in Mouse Cochlear Lgr5+ Progenitor Cells}

\author{
Yin Chen ${ }^{1 t}$, Ruiying Qiang ${ }^{2 t}$, Yuan Zhang ${ }^{2 t}$, Wei Cao ${ }^{3 t}$, Leilei Wu², Pei Jiang ${ }^{2}$, Jingru $\mathrm{Ai}^{2}$, \\ Xiangyu Ma ${ }^{2}$, Ying Dong ${ }^{2}$, Xia Gao ${ }^{1}$, He Li ${ }^{4 *}$, Ling $\mathrm{Lu}^{1 *}$, Shasha Zhang ${ }^{2 *}$ and \\ Renjie Chai2,5,6,7* \\ 1 Jiangsu Provincial Key Medical Discipline (Laboratory), Department of Otolaryngology Head and Neck Surgery, Drum Tower \\ Hospital Clinical College of Nanjing Medical University, Nanjing, China, ${ }^{2}$ State Key Laboratory of Bioelectronics, Jiangsu \\ Province High-Tech Key Laboratory for Bio-Medical Research, School of Life Sciences and Technology, Southeast \\ University, Nanjing, China, ${ }^{3}$ Department of Otorhinolaryngology, Head and Neck Surgery, The Second Hospital of Anhui \\ Medical University, Hefei, China, ${ }^{4}$ Department of Otolaryngology, The First Affiliated Hospital of Wenzhou Medical University, \\ Wenzhou, China, ${ }^{5}$ Co-innovation Center of Neuroregeneration, Nantong University, Nantong, China, ${ }^{6}$ Institute for Stem Cell \\ and Regeneration, Chinese Academy of Sciences, Beijing, China, ${ }^{7}$ Beijing Key Laboratory of Neural Regeneration \\ and Repair, Capital Medical University, Beijing, China
}

The super elongation complex (SEC) has been reported to play a key role in the proliferation and differentiation of mouse embryonic stem cells. However, the expression pattern and function of the SEC in the inner ear has not been investigated. Here, we studied the inner ear expression pattern of three key SEC components, AFF1, AFF4, and ELL3, and found that these three proteins are all expressed in both cochlear hair cells (HCs)and supporting cells (SCs). We also cultured Lgr5+ inner ear progenitors in vitro for sphere-forming assays and differentiation assays in the presence of the SEC inhibitor flavopiridol. We found that flavopiridol treatment decreased the proliferation ability of Lgr5+ progenitors, while the differentiation ability of Lgr5+ progenitors was not affected. Our results suggest that the SEC might play important roles in regulating inner ear progenitors and thus regulating $\mathrm{HC}$ regeneration. Therefore, it will be very meaningful to further investigate the detailed roles of the SEC signaling pathway in the inner ear in vivo in order to develop effective treatments for sensorineural hearing loss.

Keywords: super elongation complex (SEC), inner ear, expression, proliferation, differentiation

\section{INTRODUCTION}

Hearing loss occurs mainly due to noise exposure, aging, ototoxic drugs, and genetic factors (Sun et al., 2017). There were around 466 million people worldwide with disabling hearing loss in 2020, and the World Health Organization [WHO] (2019) estimates that by 2050 over 900 million people will have disabling hearing loss. Deafness has become a major global health problem, and sensorineural hearing loss is the most common type of hearing impairment (Youm and $\mathrm{Li}, 2018)$. However, due to the lack of effective drugs and a non-invasive method for targeted delivery of drugs to the inner ear, the treatment options for sensorineural hearing loss are 
limited (Mittal et al., 2019). Cochlear hair cells (HCs) in adult mammals lose the ability to regenerate, thus hearing deficits caused by HC loss are permanent (Warchol et al., 1993; Ryan, 2003; Cox et al., 2014; Xu et al., 2017; Chen et al., 2019). Therefore, induction of HC regeneration after injury by stimulating quiescent inner ear progenitor cells has been a main focus of auditory research in recent years.

The super elongation complex (SEC) is extremely important in the transcriptional elongation checkpoint control stage of transcription and is composed mainly of P-TEFb (positive transcription elongation factor), ELL (11-19 lysine-rich leukemia gene) family proteins, AFF (AF4/FMR2) family proteins, ENL (11-19 leukemia), AF9 (ALL1-fused gene from chromosome 9), and many other transcription factors (Luo et al., 2012b). $\mathrm{P}-\mathrm{TEFb}$ and ELL are RNA polymerase II (Pol II)-related elongation factors (Shilatifard et al., 2003). AFF family proteins act as transcriptional activators with a positive action on RNA elongation (Melko et al., 2011). ENL and AF9 are homologous, and they can connect the SEC to RNA Pol II-related factors (He et al., 2011). P-TEFb is composed of cyclin-dependent kinase 9 (CDK9) and cyclin T (CycT), and it promotes the transition into productive elongation by phosphorylating RNA polymerase II (Peng et al., 1998). It has been reported that the SEC plays an important role in regulating mouse embryonic stem cell proliferation and differentiation (Lin et al., 2011), and misregulation of the SEC leads to the uncontrolled regulation of gene expression during the differentiation of embryonic stem cells, which results in a variety of diseases such as acute lymphoblastic leukemia, cerebellar ataxia, and diffuse midline glioma (Lin et al., 2011; Dahl et al., 2020). ELL3, one of the key factors of the SEC, can protect differentiated cells from apoptosis by promoting the degradation of $\mathrm{p} 53$, enhancing the differentiation of mouse embryonic stem cells, and regulating the proliferation and survival of embryonic stem cells (Ahn et al., 2012). However, the roles of the SEC in the inner ear remain unclear.

Flavopiridol is a semi-synthetic flavonoid that has been used in the treatment of acute myeloid leukemia (Zeidner and Karp, 2015), chronic lymphocytic leukemia (Wiernik, 2016), and other chronic diseases. Flavopiridol binds directly to CDK9, which is a component of P-TEFb, and inhibits its kinase activity (Chao et al., 2000). In turn, P-TEFb, as an important component of the SEC, can activate RNA polymerase II and transcriptional elongation (Hnisz et al., 2013). Thus, the most common method for blocking SEC function is to directly inhibit CDK9 with flavopiridol (Morales and Giordano, 2016), and we used flavopiridol to inhibit the function of the SEC as previously reported (Lin et al., 2011).

Recent studies have shown that Lgr5+ supporting cells (SCs) are inner ear progenitors and that they have the ability to regenerate new $\mathrm{HCs}$ in the neonatal stage (Shi et al., 2012). The activation of $\mathrm{Wnt} / \beta$-catenin signaling and inhibition of Notch signaling can induce Lgr5+ progenitors to regenerate Myo7a+ HCs (Chai et al., 2012; Korrapati et al., 2013; Mizutari et al., 2013), and several recent studies have also shown that Lgr5+ progenitors can be regulated by many other factors and signaling pathways such as Shh, Foxg1, and Hippo (Gregorieff et al., 2015; Chen et al., 2017; Zhang et al., 2020). However, the regeneration efficiency of Lgr5+ progenitors is still very limited, which suggests that there are other factors or signaling pathways involved in the $\mathrm{HC}$ regeneration process. Because the transcription extension stage is the main stage of gene expression regulation, transcriptional regulation of developmental regulatory genes is the core link between embryonic stem cell differentiation and organ formation (Smith and Shilatifard, 2010; Levine, 2011). Therefore, we speculate that the SEC may also play important roles in cochlear progenitor cells.

Here we measured the expression of the key SEC factors AFF1, AFF4, and ELL3 in the neonatal mouse cochlea, the function of SEC inhibitor flavopiridol in House Ear Institute-Organ of Corti 1 (HEI-OC1) cell line, and we assessed the proliferation and differentiation ability of Lgr5+ progenitors after treatment with the SEC inhibitor flavopiridol. Our results suggest important roles for the SEC in Lgr5+ progenitors in vitro, and further in vivo studies need to be done to elucidate the roles of the SEC in the inner ear. These studies will form the experimental basis for using cochlear progenitors to regenerate functional HCs in order to treat patients with sensorineural hearing loss.

\section{MATERIALS AND METHODS}

\section{Experimental Animals}

Lgr5-EGFP-Ires-CreERT2 (Lgr5-EGFP) mice (Barker et al., 2007) (Jackson Laboratory, Stock No. 00887) and FVB mice used as wide-type mice were raised in a comfortable environment with suitable temperature and light and fed with standard laboratory food and water ad libitum. We are approved by the Animal Care and Use Committee of Southeast University and were consistent with the National Institutes of Health Guide for the Care and Use of Laboratory Animals. All the operations were carried out in accordance with the procedures.

\section{RNA Extraction and Reverse Transcription-Polymerase Chain Reaction}

About 20 wild-type mouse cochleae were dissected to extract total RNA, which was reverse transcribed into cDNA with the cDNA Synthesis Kit (Thermo Fisher Scientific, K1622). Gene expression was measured by reverse transcription-polymerase chain reaction (RT-PCR) with GAPDH as the endogenous reference gene. The RT-PCR conditions were as follow for a total of 35 cycles: initial denaturation at $95^{\circ} \mathrm{C}$ for $15 \mathrm{~s}$, denaturation at $95^{\circ} \mathrm{C}$ for $15 \mathrm{~s}$, annealing at $60^{\circ} \mathrm{C}$ for $60 \mathrm{~s}$, and extension at $72^{\circ} \mathrm{C}$. The primers were as follows: GAPDH: (F) $5^{\prime}$-AGG TCG GTG TGA ACG GAT TTG-3'; (R) 5'-TGT AGA CCA TGT AGT TGA GGT CA-3'; AFF1: (F) 5'-GAA GGA AAG ACG CAA CCA AGA-3'; (R) 5' TAG CTC ATC GCC TTT TGC AGT-3'; AFF4: (F) 5'-ATG AAC

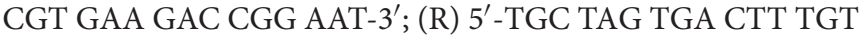
ATG GCT CA-3'; ELL3: (F) 5'-GAC CAG CCT CCT GAT GCT AAG-3'; (R) 5'-GCC ACC ATT AGT GCC CTC TTG-3'.

\section{Western Blotting}

About 10 cochleae from postnatal day (P)3 mice were dissected in order to extract proteins. GAPDH was used as the reference protein. The primary antibodies were anti-AFF1 (Sigma-Aldrich, 
\#SAB2106246), anti-AFF4 (Santa Cruz, \#sc135337), and antiELL3 (Abcam, \#ab67415). Peroxidase-conjugated goat antirabbit (Life, A-31572) and goat anti-mouse (Invitrogen, A21202) were used as the secondary antibodies. The gray levels were measured by Image-J.

\section{Cell Culture}

HEI-OC1 cells were cultured in Dulbecco's modified Eagle's medium (DMEM) with $10 \%$ fetal bovine serum and $1 \%$ ampicillin at $37^{\circ} \mathrm{C}$ and $5 \% \mathrm{CO}_{2}$. The cells were divided into two groups. The experimental group was treated with flavopiridol (AbMole M1710) at the concentration of $10 \mu \mathrm{M}$. Control cells were treated with dimethyl sulfoxide (DMSO) in the same culture medium. After 12-h culture, cells were treated with $0.25 \%$ trypsin/ethylene diamine tetraacetic acid (EDTA) and then ultrasonicated (Bioruptor ${ }^{\mathrm{TM}}$ UCD-200) for CDK9 kinase detection.

\section{Cyclin-Dependent Kinase 9 Kinase Assay}

HEI-OC1 cells with or without flavopiridol treatment were used after ultrasonication to detect the CDK9 activity by using CDK9 Cyclin K Kinase Assay kit (Promega, V4104) and ADPGlo Kinase Assay kit (Promega, V6930). To initiate the CDK9 reaction, $\mathrm{CDK} 9$ substrate $\mathrm{PDK}$ tides and adenosine triphosphate (ATP) were added into each group for $120 \mathrm{~min}$ at room temperature to produce adenosine diphosphate (ADP) according to the manufacturer's instruction (Promega, \#TM313). And then ADP-Glo Reagent was added for $40 \mathrm{~min}$ at room temperature to deplete the remaining ATP. The Kinase Detection Reagent was added to convert the ADP produced at the first step to ATP with luminescence. Finally, the luminescence was recorded by BioTek CYTATION 5 (Integration time $1 \mathrm{~s}$ ) to determine the CDK9 activity in each sample. The relative light units were calculated to represent the activity of CDK9.

\section{Isolation of Lgr5+ Progenitors via Flow Cytometry}

About 50-60 cochleae were isolated from P0 to P3 Lgr5-EGFP mice and then treated with $0.125 \%$ trypsin/EDTA (Invitrogen, $25200114)$ at $37^{\circ} \mathrm{C}$. Trypsin inhibitor $(10 \mathrm{mg} / \mathrm{ml}$, Worthington Biochem) was added after $10 \mathrm{~min}$ to terminate the reaction. The trypsinized cochleae were pipetted up and down 80-100 times to obtain single cells, and the cells were then filtered through a $40 \mu \mathrm{M}$ cell strainer (BD Biosciences, 352340). Dissociated cells were sorted on a flow cell sorter (BD FACS Aria III). The EGFP+ cells were collected as Lgr5+ progenitors for further in vitro cell culture experiments.

\section{Sphere-Forming Assay and Differentiation Assay}

Sorted Lgr5+ cells were cultured in DMEM/F12 medium at a density of 2 cells/ $\mu$ l (200 cells per well) for 5 days for sphere forming. The formula of DMEM/F12 medium was the same in previous study (Zhang et al., 2020). Spheres were identified with the Live Cell Imaging System and quantified using Image J. For differentiation, cells were cultured in the DMEM/F12 medium described above at a density of 20 cells/ $\mu 1$ (2,000 cells per well) for 10 days. EdU [10 $\mu \mathrm{M}$ (Invitrogen, C10420)] was added to label proliferating cells from day 4 to day 7. Flavopiridol (AbMole, M1710) was added to the experimental group from day 1 to day 10 at a concentration of $10 \mu \mathrm{M}$, while DMSO was added to the control group. Differentiated neurospheres were analyzed by immunofluorescent staining.

\section{Immunofluorescent Staining}

The cochleae were dissected in cold Hanks Balanced Salt Solution (HBSS) in order to prevent protein degradation and then fixed with $4 \%$ paraformaldehyde (PFA) for $1 \mathrm{~h}$ at room temperature. In vitro cultured neurospheres were also fixed with $4 \%$ PFA for $1 \mathrm{~h}$ at room temperature. After washing with phosphate buffered saline with tween (PBST) three times, the cochleae or neurospheres were blocked with blocking solution for $1 \mathrm{~h}$ at room temperature and then incubated overnight at $4^{\circ} \mathrm{C}$ with primary antibodies. The primary antibodies used were anti-Myosin7a (Myo7a; Proteus Bioscience, \#25-6790; 1:1,000 dilution), antiSox2 (1:400 dilution), anti-AFF1 (1:400 dilution), anti-AFF4 (1:50 dilution), and anti-ELL3 (1:400 dilution). After washing again three times, the cochleae or neurospheres were further incubated with secondary antibodies (Invitrogen, A21131, A21124) diluted 1:400 in PBT2 for $1 \mathrm{~h}$ at room temperature. After washing three times, the cochleae or neurospheres were mounted on slides with anti-fade fluorescence mounting medium (DAKO, S3023). Images were captured by Zeiss LSM 710 confocal microscope and analyzed by Image J software.

\section{Tissue Embedment}

The P40 temporal bones were dissected and put in 4\% PFA to be shaken for $1-2 \mathrm{~h}$ and sit overnight at $4^{\circ} \mathrm{C}$. Later, the temporal bones were put in 0.5 M EDTA for decalcification for 2 days. After washing with PBST three times, the temporal bones were transferred into $15 \%$ sucrose solution, vacuum for $1 \mathrm{~h}, 4^{\circ} \mathrm{C}$ overnight. Afterward, the temporal bones were put in $20 \%$ sucrose solution, vacuum for $1 \mathrm{~h}$, then transferred to $30 \%$ sucrose solution, vacuum $1 \mathrm{~h}, 4^{\circ} \mathrm{C}$ overnight. Then, the temporal bones were put into a $1: 1$ solution of $30 \%$ sucrose in optimum cutting temperature (OCT) medium (Sakura 4583), vacuum for $1 \mathrm{~h}$, overnight at $4^{\circ} \mathrm{C}$. The following day, temporal bones were put in a 3:7 solution of $30 \%$ sucrose in OCT medium, vacuum for $1 \mathrm{~h}$, then the 3:17 solution of $30 \%$ sucrose in OCT medium, vacuum for $1 \mathrm{~h}$, and lastly the $100 \%$ OCT medium (adjust position as round window and ellipse window are toward on the ground), vacuum for $1 \mathrm{~h}, 4^{\circ} \mathrm{C}$ overnight. For the last step, the temporal bones were put in $100 \%$ OCT into vacuum for $1 \mathrm{~h}$ adjust position, then in cryostat (Microm HM525) for a 20-min quick-freeze, and restored in $-80^{\circ} \mathrm{C}$. For slicing, adjust the cryostat half an hour in advance; secondly, adjust the blade temperature and internal temperature to $-20^{\circ} \mathrm{C}$. The selected sections were stained using the method described above.

\section{Statistical Analysis}

All the data in this research are presented as means \pm SEM, and all experiments were repeated at least three times. All statistical analyses were performed in GraphPad Prism 5. P-values were calculated using a two-tailed, unpaired Student's $t$-test, and a $p$-value $<0.05$ was considered statistically significant. 


\section{RESULTS}

\section{AFF1, AFF4, and ELL3 Are Expressed in the Cochlea}

We first measured the expression of the three key SEC subunits AFF1, AFF4, and ELL3 by RT-PCR (Figure 1A) and Western blotting analysis (Figures 1B,C), and we found that AFF1, AFF4, and ELL3 were all highly expressed in the cochlea. Moreover, we measured the expression of AFF1, AFF4, and ELL3 in Lgr5+ cells (Figure 1D), and we found those three expressions in both cochlea and Lgr5+ cells were similar. We further studied the expression pattern from both the obverse and lateral sides of AFF1, AFF4, and ELL3 in the cochlea of P3 and P40 mice and found that AFF1, AFF4, and ELL3 were all expressed in the cochlear HCs and SCs (Figures 2A-D). However, the immunostaining intensities of these three subunits in the SCs were weaker than in the HCs.

\section{Flavopiridol Treatment Inhibited the Activity of Cyclin-Dependent Kinase 9 in House Ear Institute-Organ of Corti 1 Cells}

Flavopiridol has been reported to be an inhibitor of CDK9 which is an indispensable part of SEC and the low level of its kinase activity prevents the recruitment of other elongation factors in
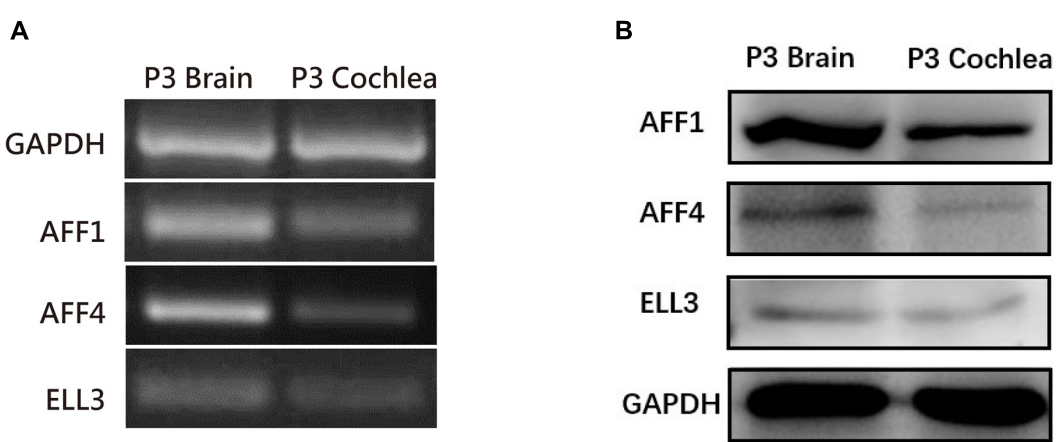

C

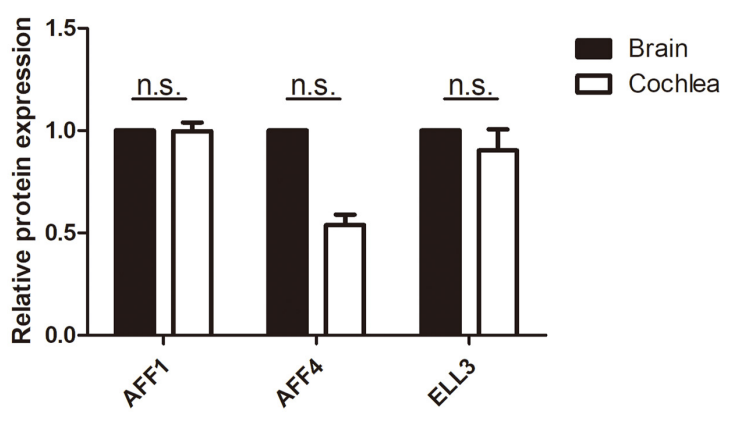

D

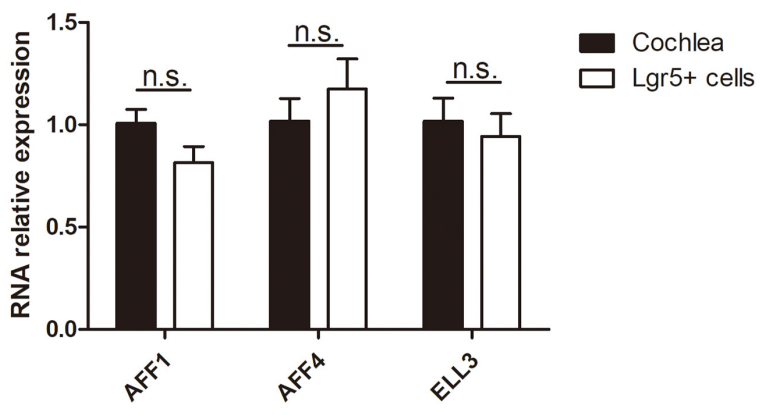

FIGURE 1 | The expression of AFF1, AFF4, and ELL3 in the neonatal mouse cochlea. (A,B) The mRNA and protein expression of AFF1, AFF4, and ELL3 in P3 mouse cochleae were detected by RT-PCR (A) and western blotting (B), respectively. (C) The gray levels comparison of western blot. (D) The mRNA expression of AFF1, AFF4, and ELL3 in Lgr5+ cells. Brain samples of P3 mice were used as the positive control, and GAPDH was used as the internal reference. n.s., not significant. 


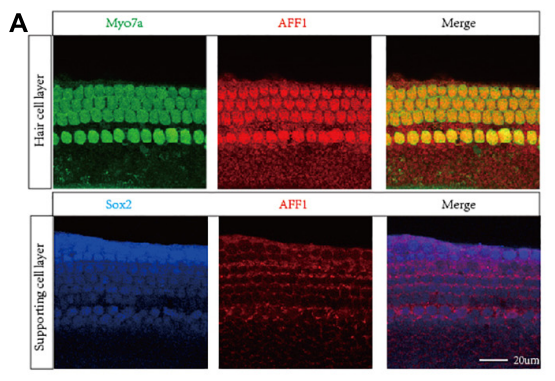

B

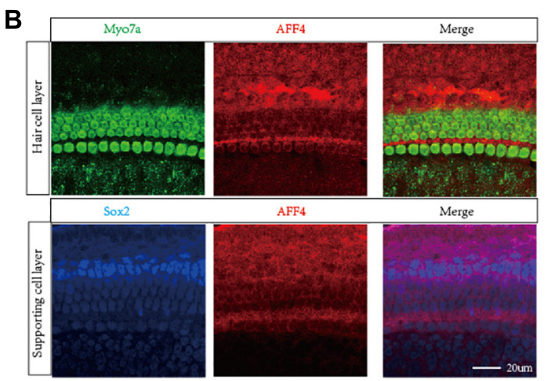

C

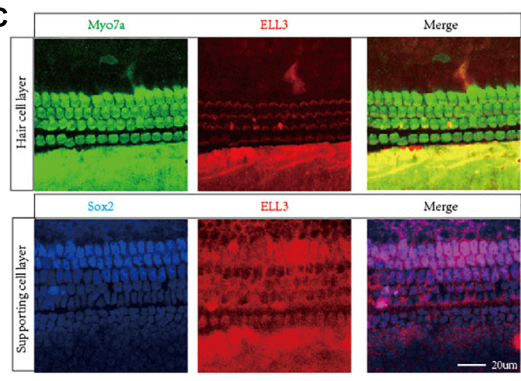

D

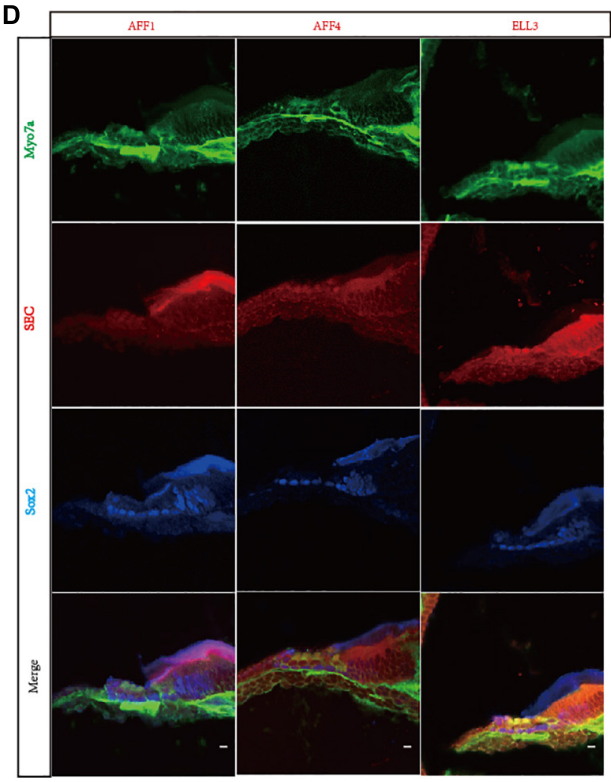

FIGURE 2 | Immunofluorescent staining of AFF1, AFF4, and ELL3 in the mouse cochlea. (A-C) The whole-mount basilar membrane of P3 was immunostained by antibodies against AFF1 (A), AFF4 (B), and ELL3 (C). (D) Frozen sections of $\mathrm{P} 40$ were immunostained by antibodies against AFF1, AFF4, and ELL3. Myo7a and Sox2 were used to label hair cells (HCs) and supporting cells (SCs), respectively. Scale bar $=20 \mu \mathrm{M}$.
SEC (Peng et al., 1998). However, the function of flavopiridol has not been verified in inner ear. Here, we used ADP-Glo Kinase Assay to detect the activity of CDK9. After the kinase reaction, the remaining ATP was depleted and the ADP was converted to luminescent ATP (Figure 3A). HEI-OC1 cells were cultured for 3 days, and then treated by $10 \mu \mathrm{M}$ flavopiridol which was diluted in the culture medium for $12 \mathrm{~h}$. Images of cells were taken before and after flavopiridol treatment (Figure 3B). After flavopiridol treatment, the number and diameter of cells visually decrease in comparison with the control group with no change in shapes. The luminescent ATP was recorded and the relative light units was calculated to represent the activity of CDK9 (Figure 3C). The results showed that flavopiridol could also function as the CDK9 inhibitor to inhibit SEC activity in HEI-OC1 cells.

\section{Flavopiridol Treatment Decreased the Sphere-Forming Ability of Lgr5+ Progenitors in vitro}

Flavopiridol was previously used to inhibit SEC transcription activity (Lin et al., 2011). Here we also used flavopiridol to inhibit SEC activity in Lgr5+ progenitors in order to determine whether the SEC plays roles in the proliferation and differentiation ability of Lgr5+ progenitors. In order to determine the effect of the SEC on the sphere-forming ability of Lgr5+ progenitors, Lgr5+ cells were isolated from Lgr5-EGFP mice by flow cytometry and then cultured in vitro for 5 days to form spheres with or without $10 \mu \mathrm{M}$ flavopiridol treatment (Figures 4A,B). The flavopiridol treatment decreased both the number (Figure $4 \mathrm{C}$ ) and diameter of the spheres (Figure 4D), which suggested that inhibition of the SEC could decrease the sphere-forming ability and proliferation ability of Lgr5+ progenitors in vitro.

\section{No Difference Was Observed in the Differentiation Assay After Flavopiridol Treatment}

In order to further evaluate the effect of the SEC on the differentiation ability of Lgr5+ progenitors, we isolated Lgr5+ cells by flow cytometry and cultured them in vitro for the differentiation assay with or without $10 \mu \mathrm{M}$ flavopiridol treatment (Figure 5A). The cells were immunostained with Myo7a, EdU, and DAPI (Figure 5B), and the Myo7a + cells and EdU+ cells inside and outside the colonies were quantified. There were more EdU+ cells in the flavopiridol treatment group than in the control group (Figure 5C), while the numbers of Myo7a+ cells were almost the same in the flavopiridol treatment group and the control group (Figure 5D). These results suggested that inhibition of the SEC did not affect the differentiation ability of Lgr5+ cells in vitro.

In summary, our results showed that the key SEC factors AFF1, AFF4, and ELL3 are all highly expressed in the cochlea. And we verified that flavopiridol could inhibit SEC by inhibiting CDK9 activity in HEI-OC1 cell line. We used flavopiridol as an SEC inhibitor to investigate the effect of the SEC on the proliferation and differentiation ability of Lgr5+ progenitors and found that the number and diameter of spheres of Lgr5+ progenitors were both decreased after SEC inhibitor treatment, 


\section{A}

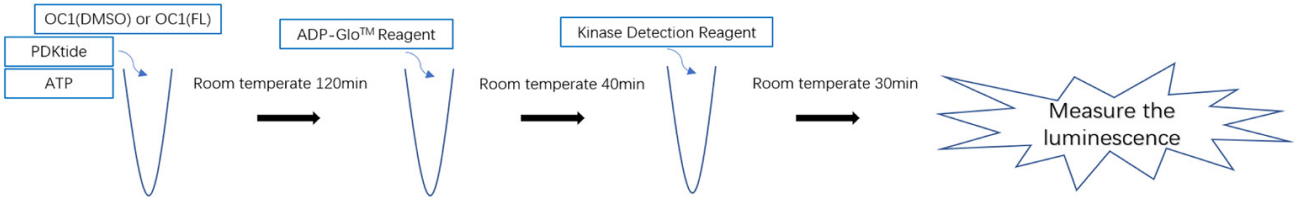

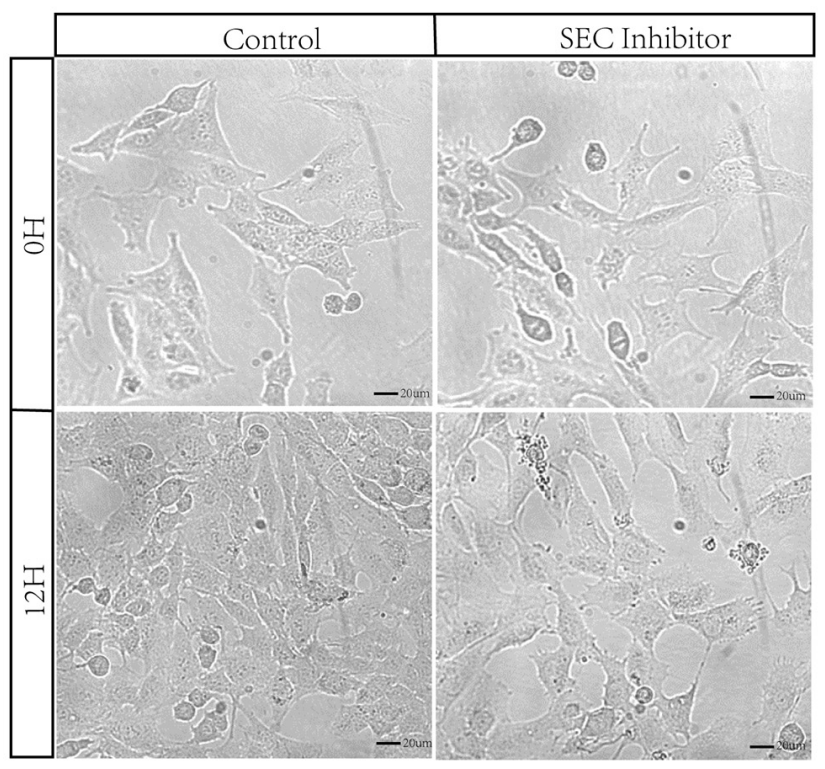

C

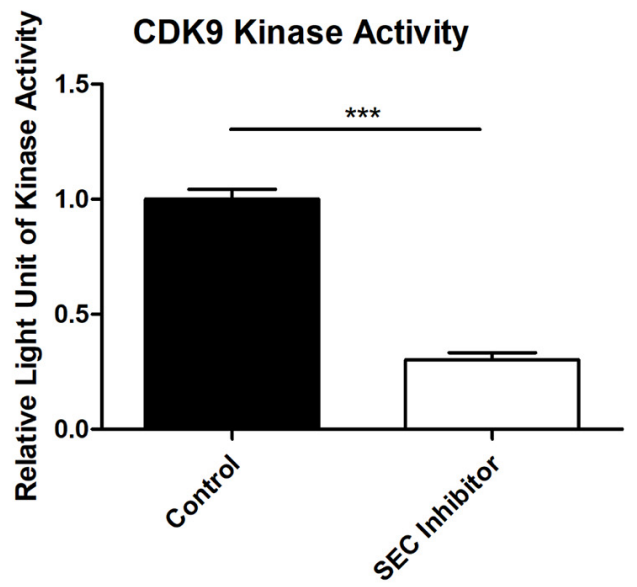

FIGURE 3 | The activity of cyclin-dependent kinase 9 (CDK9) in HEI-OC1 cells before and after Flavopiridol treatment. (A) HEI-OC1 cells after ultrasonication were incubated with CDK9 substrate PDKtides and ATP for 120 min at room temperature. The ADP which was consumed during CDK9 kinase assay was converted to ATP and could be quantitated by luciferase assay. (B) Images of HEI-OC1 cells before and after $10 \mu \mathrm{M}$ Flavopiridol treatment. DMSO was added as control treatment in the Control group. Scale bar $=20 \mu \mathrm{M}$. (C) Fold change of CDK9 kinase activity in HEl-OC1 cells with or without Flavopiridol treatment. $n=3,{ }^{* \star *} p<0.001$.

while the differentiation ability of Lgr5+ progenitors was not affected. Therefore, the SEC appears to promote the proliferation ability of Lgr5+ progenitors, but not their differentiation ability.

\section{DISCUSSION}

Irreversible damage to HCs in the mammalian cochlea is the main cause of sensorineural hearing loss. Previous studies have reported that Lgr5+ cells are cochlear progenitors with the ability to regenerate HCs (Shi et al., 2012), and it has been documented that the SEC plays an important role in the process of gene transcription and extension and that it is necessary for the differentiation of mouse embryonic stem cells (Lin et al., 2011). Therefore, we speculated that the SEC also plays an important role in the proliferation and differentiation of mouse cochlear progenitors. Besides, it has also been reported that AFF proteins and ELL proteins increase the diversity and regulatory potential of the SEC family in mammals (Luo et al., 2012a). Furthermore, AFF1 and AFF4 are the backbones of the SEC (Mück et al., 2016) and ELL3 has the ability to associate with other translocation partners of the SEC (Lin et al., 2011). Thus we chose to investigate the expression of AFF1, AFF4, and ELL3 in the inner ear. Our results showed that AFF1, AFF4, and ELL3, were all highly expressed in the cochlea and that the SEC inhibitor flavopiridol could induce the proliferation of Lgr5+ progenitors in vitro, but not their differentiation. This study thus provides an experimental foundation for the clinical application of $\mathrm{HC}$ regeneration for treating hearing loss.

The SEC is known to be widely expressed in most tissues, and it plays important roles during development (Kapushesky et al., 2010). However, its expression in the inner ear has not been studied. We found that AFF1, AFF4, and ELL3 were highly expressed in the cochlea, and this suggests that the SEC functions in the inner ear. Furthermore, we found that AFF1, AFF4, and ELL3 were all expressed in the cochlear HCs and SCs by immunostaining, but the expression of SEC proteins in SCs was lower than that in HCs. In addition to its role in Lgr5+ progenitors studied in our research, the SEC likely plays an essential role in cochlear HCs. However, due to the lack of studies of the SEC in the inner ear and the lack of mouse models, the specific role of the SEC in HCs awaits further study.

Flavopiridol, a potent inhibitor of CDK9, is reported to inhibit transcription (Blagosklonny, 2004; Lee and Zeidner, 2019). CDKs combine with cyclins to play important roles in transcription and stem cell self-renewal (Lim and Kaldis, 2013), and P-TEFb (composed of CDK9 and CycT) is an indispensable part of the SEC that phosphorylates RNA polymerase II and thus 
A
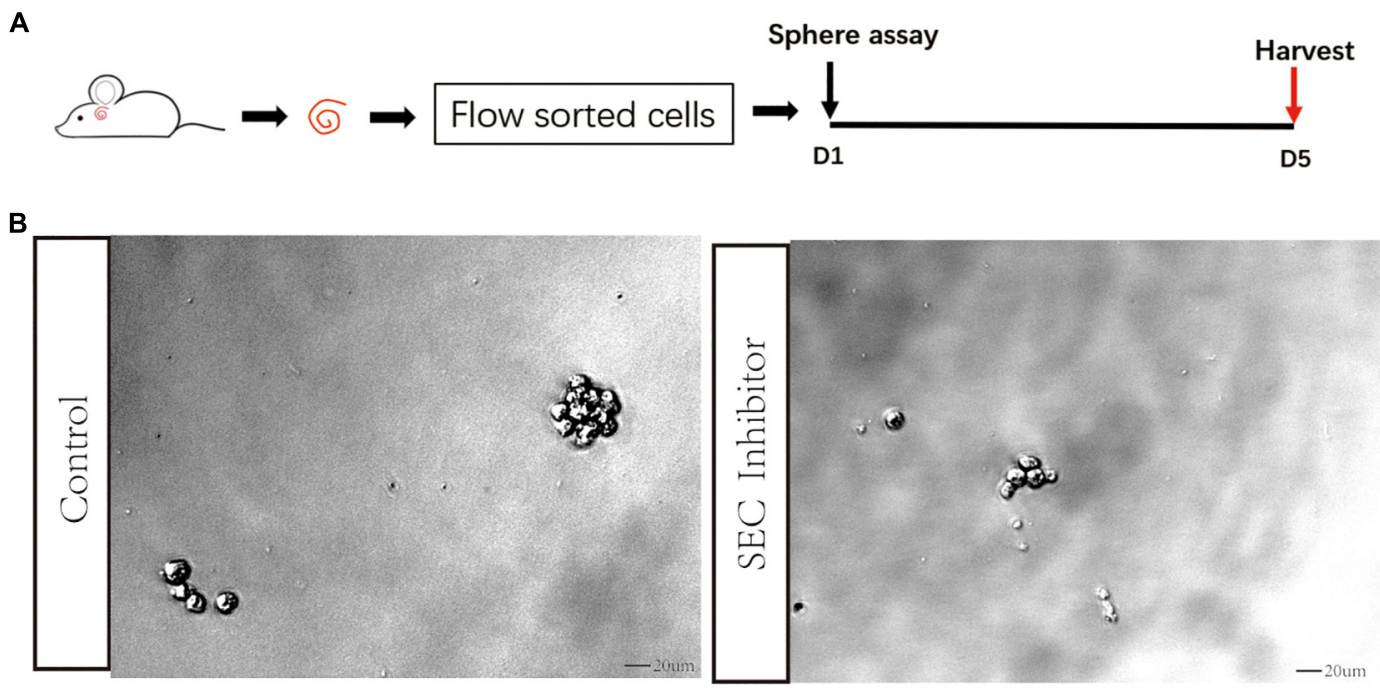

C
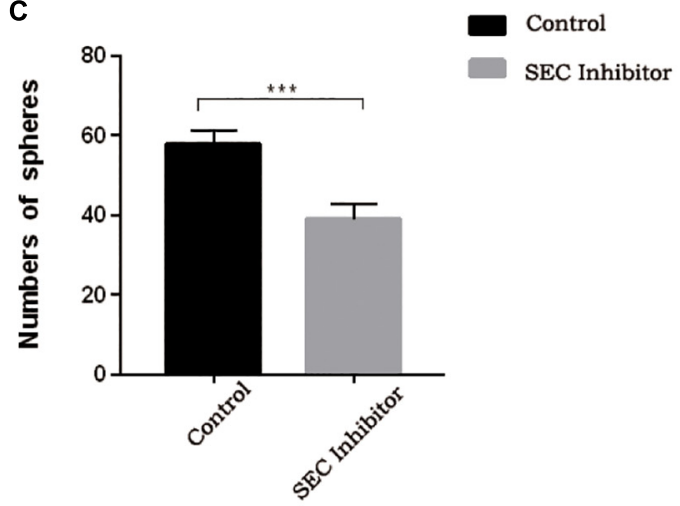

D

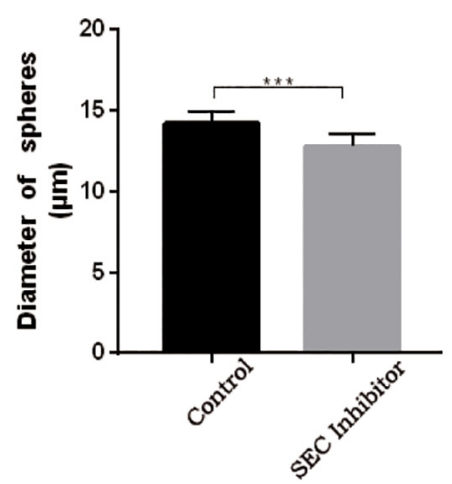

Control

SEC Inhibitor

FIGURE 4 | The sphere-forming assay of Lgr5+ progenitors after inhibition of the super elongation complex (SEC). (A) Schematic of the sphere-forming assay. The cochleae of neonatal Lgr5-EGFP-CreER mice were trypsinized for FAC sorting, and the sorted Lgr5+ progenitors were cultured in vitro for 5 days to form spheres with or without $10 \mu \mathrm{M}$ flavopiridol treatment. (B) The spheres (indicated by black arrows) formed by Lgr5+ progenitors with $10 \mu \mathrm{M}$ flavopiridol added as the SEC inhibitor. DMSO was added as control treatment in the Control group. Scale bar $=20 \mu$ M. (C,D) Quantification of the sphere number per well (C) and the sphere diameter (D). $n=3 .{ }^{\star \star \star} P<0.001$.

activates transcription elongation of important genes involved in cell proliferation and survival (Zhu et al., 1997; Zeidner and Karp, 2015). Flavopiridol can inhibit the function of the SEC by inactivating CDK9 (Chao and Price, 2001), and thus we chose flavopiridol as the SEC inhibitor as previously reported (Lin et al., 2011).

In addition, our results suggest that inhibition of the SEC by flavopiridol could reduce the sphere-forming ability and proliferating cell number of Lgr5+ progenitors in the differentiation assay, which is consistent with previous reports that AFF1 promotes leukemia cell proliferation (Fioretti et al., 2019), that AFF4 enhances the sphere-forming capacity and tumor-initiation capacity in head and neck squamous cell carcinoma (Deng et al., 2018), and that ELL3 stimulates the proliferation and stem cell properties of breast cancer cells (Ahn et al., 2013).

ELL3 has also been shown to promote the differentiation of mouse embryonic stem cells by regulating the epithelial-mesenchymal transition and apoptosis (Ahn et al., 2012), and the overexpression of AFF1 impairs the differentiation of mesenchymal stem cells, while overexpression of AFF4 enhances their differentiation (Zhou et al., 2017). In our results, inhibition of the SEC did not affect the differentiation ability of Lgr5+ progenitors in vitro, which might be because of the different regulatory roles of these three proteins in cell differentiation.

The different roles of SEC in the proliferation and differentiation ability of Lgr5 progenitors might have been expected. SEC was first systematically studied for being associated with infant acute lymphoblastic and mixed lineage leukemia (Lin et al., 2010). Then it has since been studied in other neoplastic models (Hashizume et al., 2014; Narita et al., 2017; Liang et al., 2018). While transcriptional regulation is a complex process, it has been sure that the role of SEC is essential in transcriptional elongation. The SEC incorporates the CDK9 to promote rapid transcriptional elongation facilitates cell growth 

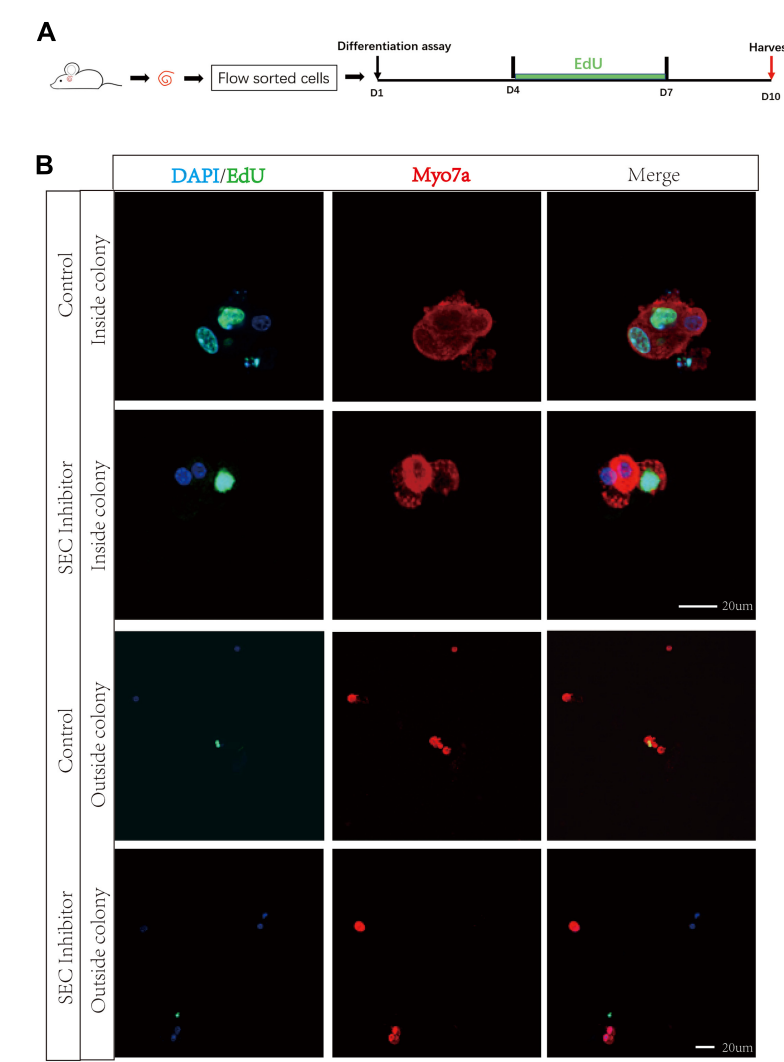

C

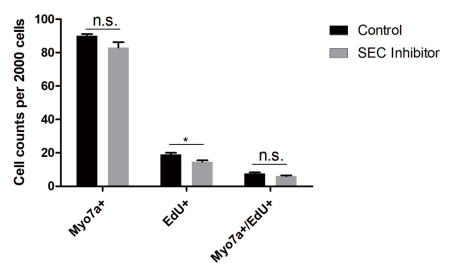

D

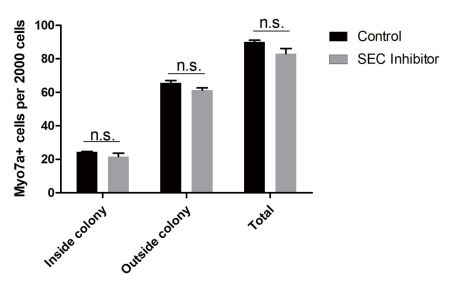

FIGURE $\mathbf{5}$ | The differentiation assay of Lgr5+ cells after inhibition of the super elongation complex (SEC). (A) Schematic of the differentiation assay. The cochleae of neonatal Lgr5-EGFP-CreER mice were trypsinized for FAC sorting, and the sorted Lgr5+ progenitors were cultured in vitro for 10 days with or without $10 \mu \mathrm{M}$ flavopiridol. EdU was added during day 4-7 to label proliferating cells. (B) Immunofluorescence images of colonies and single cells formed from Lgr5+ progenitors with $10 \mu \mathrm{M}$ flavopiridol added as the SEC inhibitor. DMSO was used as the control treatment in the Control group. Myo7a and Sox2 were used to label hair cells ( $\mathrm{HCs}$ ) and supporting cells (SCs), respectively, and EdU was used to label proliferating cells. Scale bar $=20 \mu \mathrm{M}$. (C,D) Quantification of the total numbers of Myo7a+ and EdU+ cells (C) and the number of Myo7a+ cells inside and outside the colonies (D). $n=3$. n.s., not significant. ${ }^{*} P<0.05$.
(Dahl et al., 2020). However, speaking of the differentiation ability of SEC, totally different results were found concerning the different components of this complex. Our paper first studied the differentiation ability of the total complex and found that the whole SEC cannot change the differentiation in Lgr5 progenitors. There is a certain possibility that this finding may also apply to other stem cells. The detailed mechanisms behind this need further exploration.

\section{CONCLUSION}

In conclusion, we show here that the SEC is expressed in cochlear HCs and SCs in neonatal mice and that the SEC can induce the proliferation ability of Lgr5+ progenitors but not their differentiation. Our study thus provides new candidates for regulating inner ear progenitor cells as a step toward $\mathrm{HC}$ regeneration.

\section{DATA AVAILABILITY STATEMENT}

The raw data supporting the conclusions of this article will be made available by the authors, without undue reservation.

\section{ETHICS STATEMENT}

The animal study was reviewed and approved by the Animal Care and Use Committee of Southeast University, the National Institutes of Health, and the Guide for the Care and Use of Laboratory Animals.

\section{AUTHOR CONTRIBUTIONS}

YC, HL, LL, SZ, and RC conceived and designed the experiments and wrote the manuscript. YC, RQ, YZ, WC, YD, LW, PJ, JA, and XM performed the experiments. YC, RQ, YZ, WC, XG, LL, $\mathrm{SZ}$, and RC analyzed the data. All authors read and approved the final manuscript.

\section{FUNDING}

This work was supported by grants from the National Key R\&D Program of China (No. 2017YFA0103903), the Strategic Priority Research Program of the Chinese Academy of Sciences (XDA16010303), the National Natural Science Foundation of China (Nos. 82171149, 81970882, 81970892, and 81700913), the Natural Science Foundation of Jiangsu Province (BE2019711, BE2018605, and BK20190062), the Jiangsu Provincial Medical Youth Talent of the Project of Invigorating Health Care through Science, Technology and Education (QNRC2016002), the Fundamental Research Funds for the Central Universities for the Support Program of Zhishan Youth Scholars of Southeast University (2242021R41136), and the Zhejiang Provincial Natural Science Foundation of China (LY19H130004). 


\section{REFERENCES}

Ahn, H.-J., Cha, Y., Moon, S.-H., Jung, J.-E., and Park, K.-S. (2012). Ell3 enhances differentiation of mouse embryonic stem cells by regulating epithelialmesenchymal transition and apoptosis. PLoS One 7:e40293. doi: 10.1371/ journal.pone.0040293

Ahn, H.-J., Kim, G., and Park, K.-S. (2013). Ell3 stimulates proliferation, drug resistance, and cancer stem cell properties of breast cancer cells via a MEK/ERK-dependent signaling pathway. Biochem. Biophys. Res. Commun. 437, 557-564. doi: 10.1016/j.bbrc.2013.06.114

Barker, N., van Es, J. H., Kuipers, J., Kujala, P., van den Born, M., Cozijnsen, M., et al. (2007). Identification of stem cells in small intestine and colon by marker gene Lgr5. Nature 449, 1003-1007.

Blagosklonny, M. V. (2004). Flavopiridol, an inhibitor of transcription: implications, problems and solutions. Cell Cycle 3, 1537-1542. doi: 10.4161/cc. 3.12.1278

Chai, R., Kuo, B., Wang, T., Liaw, E. J., Xia, A., Jan, T. A., et al. (2012). Wnt signaling induces proliferation of sensory precursors in the postnatal mouse cochlea. Proc. Natl. Acad. Sci. U.S.A. 109, 8167-8172. doi: 10.1073/pnas. 1202774109

Chao, S. H., Fujinaga, K., Marion, J. E., Taube, R., Sausville, E. A., Senderowicz, A. M., et al. (2000). Flavopiridol inhibits P-TEFb and blocks HIV-1 replication. J. Biol. Chem. 275, 28345-28348. doi: 10.1074/jbc.C000446200

Chao, S. H., and Price, D. H. (2001). Flavopiridol inactivates P-TEFb and blocks most RNA polymerase II transcription in vivo. J. Biol. Chem. 276, 31793-31799. doi: 10.1074/jbc.M102306200

Chen, Y., Lu, X., Guo, L., Ni, W., Zhang, Y., Zhao, L., et al. (2017). Hedgehog signaling promotes the proliferation and subsequent hair cell formation of progenitor cells in the neonatal mouse cochlea. Front. Mol. Neurosci. 10:426. doi: 10.3389/fnmol.2017.00426

Chen, Y., Zhang, S., Chai, R., and Li, H. (2019). Hair cell regeneration. Adv. Exper. Med. Biol. 1130, 1-16. doi: 10.1007/978-981-13-6123-4_1

Cox, B. C., Chai, R., Lenoir, A., Liu, Z., Zhang, L., Nguyen, D.-H., et al. (2014). Spontaneous hair cell regeneration in the neonatal mouse cochlea in vivo. Development 141, 816-829. doi: 10.1242/dev.103036

Dahl, N. A., Danis, E., Balakrishnan, I., Wang, D., Pierce, A., Walker, F. M., et al. (2020). Super elongation complex as a targetable dependency in diffuse midline glioma. Cell Rep. 31:107485. doi: 10.1016/j.celrep.2020.03.049

Deng, P., Wang, J., Zhang, X., Wu, X., Ji, N., Li, J., et al. (2018). AFF4 promotes tumorigenesis and tumor-initiation capacity of head and neck squamous cell carcinoma cells by regulating SOX2. Carcinogenesis 39, 937-947. doi: 10.1093/ carcin/bgy046

Fioretti, T., Cevenini, A., Zanobio, M., Raia, M., Sarnataro, D., Salvatore, F., et al. (2019). Crosstalk between 14-3-30 and AF4 enhances MLL-AF4 activity and promotes leukemia cell proliferation. Cell Oncol. 42, 829-845. doi: 10.1007/ s13402-019-00468-6

Gregorieff, A., Liu, Y., Inanlou, M. R., Khomchuk, Y., and Wrana, J. L. (2015). Yapdependent reprogramming of Lgr5(+) stem cells drives intestinal regeneration and cancer. Nature 526, 715-718. doi: 10.1038/nature 15382

Hashizume, R., Andor, N., Ihara, Y., Lerner, R., Gan, H., Chen, X., et al. (2014). Pharmacologic inhibition of histone demethylation as a therapy for pediatric brainstem glioma. Nat. Med. 20, 1394-1396. doi: 10.1038/nm.3716

He, N., Chan, C. K., Sobhian, B., Chou, S., Xue, Y., Liu, M., et al. (2011). Human Polymerase-Associated Factor complex (PAFc) connects the Super Elongation Complex (SEC) to RNA polymerase II on chromatin. Proc. Natl. Acad. Sci. U.S.A. 108, E636-E645. doi: 10.1073/pnas.1107107108

Hnisz, D., Abraham, B. J., Lee, T. I., Lau, A., Saint-André, V., Sigova, A. A., et al. (2013). Super-enhancers in the control of cell identity and disease. Cell 155, 934-947. doi: 10.1016/j.cell.2013.09.053

Kapushesky, M., Emam, I., Holloway, E., Kurnosov, P., Zorin, A., Malone, J., et al. (2010). Gene expression atlas at the European bioinformatics institute. Nucleic Acids Res. 38, D690-D698. doi: 10.1093/nar/gkp936

Korrapati, S., Roux, I., Glowatzki, E., and Doetzlhofer, A. (2013). Notch signaling limits supporting cell plasticity in the hair cell-damaged early postnatal murine cochlea. PLoS One 8:e73276. doi: 10.1371/journal.pone.00 73276

Lee, D. J., and Zeidner, J. F. (2019). Cyclin-dependent kinase (CDK) 9 and $4 / 6$ inhibitors in acute myeloid leukemia (AML): a promising therapeutic approach. Expert Opin. Investig. Drugs 28, 989-1001. doi: 10.1080/13543784. 2019.1678583

Levine, M. (2011). Paused RNA polymerase II as a developmental checkpoint. Cell 145, 502-511. doi: 10.1016/j.cell.2011.04.021

Liang, K., Smith, E. R., Aoi, Y., Stoltz, K. L., Katagi, H., Woodfin, A. R., et al. (2018). Targeting processive transcription elongation via SEC disruption for MYC-induced cancer therapy. Cell 175, 766-779.e717. doi: 10.1016/j.cell.2018. 09.027

Lim, S., and Kaldis, P. (2013). Cdks, cyclins and CKIs: roles beyond cell cycle regulation. Development 140, 3079-3093. doi: 10.1242/dev.091744

Lin, C., Garrett, A. S., De Kumar, B., Smith, E. R., Gogol, M., Seidel, C., et al. (2011). Dynamic transcriptional events in embryonic stem cells mediated by the super elongation complex (SEC). Genes Dev. 25, 1486-1498. doi: 10.1101/ gad.2059211

Lin, C., Smith, E. R., Takahashi, H., Lai, K. C., Martin-Brown, S., Florens, L., et al. (2010). AFF4, a component of the ELL/P-TEFb elongation complex and a shared subunit of MLL chimeras, can link transcription elongation to leukemia. Mol. Cell 37, 429-437. doi: 10.1016/j.molcel.2010.01.026

Luo, Z., Lin, C., and Shilatifard, A. (2012b). The super elongation complex (SEC) family in transcriptional control. Nat. Rev. Mol. Cell Biol. 13, 543-547. doi: $10.1038 / \mathrm{nrm} 3417$

Luo, Z., Lin, C., Guest, E., Garrett, A. S., Mohaghegh, N., Swanson, S., et al. (2012a). The super elongation complex family of RNA polymerase II elongation factors: gene target specificity and transcriptional output. Mol. Cell Biol. 32, 2608-2617. doi: $10.1128 / \mathrm{mcb} .00182-12$

Melko, M., Douguet, D., Bensaid, M., Zongaro, S., Verheggen, C., Gecz, J., et al. (2011). Functional characterization of the AFF (AF4/FMR2) family of RNAbinding proteins: insights into the molecular pathology of FRAXE intellectual disability. Hum. Mol. Genet. 20, 1873-1885. doi: 10.1093/hmg/ddr069

Mittal, R., Pena, S. A., Zhu, A., Eshraghi, N., Fesharaki, A., Horesh, E. J., et al. (2019). Nanoparticle-based drug delivery in the inner ear: current challenges, limitations and opportunities. Artific. Cells Nanomed. Biotechnol. 47, 1312 1320. doi: 10.1080/21691401.2019.1573182

Mizutari, K., Fujioka, M., Hosoya, M., Bramhall, N., Okano, H. J., Okano, H., et al. (2013). Notch inhibition induces cochlear hair cell regeneration and recovery of hearing after acoustic trauma. Neuron 77, 58-69. doi: 10.1016/j.neuron.2012. 10.032

Morales, F., and Giordano, A. (2016). Overview of CDK9 as a target in cancer research. Cell Cycle 15, 519-527. doi: 10.1080/15384101.2016.1138186

Mück, F., Bracharz, S., and Marschalek, R. (2016). DDX6 transfers P-TEFb kinase to the AF4/AF4N (AFF1) super elongation complex. Am. J. Blood Res. 6, 28-45.

Narita, T., Ishida, T., Ito, A., Masaki, A., Kinoshita, S., Suzuki, S., et al. (2017). Cyclin-dependent kinase 9 is a novel specific molecular target in adult T-cell leukemia/lymphoma. Blood 130, 1114-1124. doi: 10.1182/blood-201609-741983

Peng, J., Zhu, Y., Milton, J. T., and Price, D. H. (1998). Identification of multiple cyclin subunits of human P-TEFb. Genes Dev. 12, 755-762. doi: 10.1101/gad.12. 5.755

Ryan, A. F. (2003). The cell cycle and the development and regeneration of hair cells. Curr. Top. Dev. Biol. 57, 449-466.

Shi, F., Kempfle, J. S., and Edge, A. S. B. (2012). Wnt-responsive Lgr5-expressing stem cells are hair cell progenitors in the cochlea. J. Neurosci. 32, 9639-9648. doi: 10.1523/JNEUROSCI.1064-12.2012

Shilatifard, A., Conaway, R. C., and Conaway, J. W. (2003). The RNA polymerase II elongation complex. Annu. Rev. Biochem. 72, 693-715. doi: 10.1146/annurev. biochem.72.121801.161551

Smith, E., and Shilatifard, A. (2010). The chromatin signaling pathway: diverse mechanisms of recruitment of histone-modifying enzymes and varied biological outcomes. Mol. Cell 40, 689-701. doi: 10.1016/j.molcel.2010.11.031

Sun, W., Yang, S., Liu, K., and Salvi, R. J. (2017). Hearing loss and auditory plasticity. Hear. Res. 347, 1-2. doi: 10.1016/j.heares.2017.03.010

Warchol, M. E., Lambert, P. R., Goldstein, B. J., Forge, A., and Corwin, J. T. (1993). Regenerative proliferation in inner ear sensory epithelia from adult guinea pigs and humans. Science 259, 1619-1622.

Wiernik, P. H. (2016). Alvocidib (flavopiridol) for the treatment of chronic lymphocytic leukemia. Expert Opin. Investig. Drugs 25, 729-734. doi: 10.1517/ 13543784.2016.1169273 
World Health Organization [WHO] (2019). Fact Sheet. Deafness and Hearing Impairment. Available online at http://www.who.int/mediacentre/factsheets/ fs300/en/index.html (accessed March 20, 2019).

Xu, J., Ueno, H., Xu, C. Y., Chen, B., Weissman, I. L., and Xu, P.-X. (2017). Identification of mouse cochlear progenitors that develop hair and supporting cells in the organ of Corti. Nat. Commun. 8:15046. doi: 10.1038/ncomms 15046

Youm, I., and Li, W. (2018). Cochlear hair cell regeneration: an emerging opportunity to cure noise-induced sensorineural hearing loss. Drug Discov. Today 23, 1564-1569. doi: 10.1016/j.drudis.2018.05.001

Zeidner, J. F., and Karp, J. E. (2015). Clinical activity of alvocidib (flavopiridol) in acute myeloid leukemia. Leuk. Res. 39, 1312-1318. doi: 10.1016/j.leukres.2015. 10.010

Zhang, S., Zhang, Y., Dong, Y., Guo, L., Zhang, Z., Shao, B., et al. (2020). Knockdown of Foxg1 in supporting cells increases the trans-differentiation of supporting cells into hair cells in the neonatal mouse cochlea. Cell Mol. Life Sci. 77, 1401-1419. doi: 10.1007/s00018-019-03291-2

Zhou, C.-C., Xiong, Q.-C., Zhu, X.-X., Du, W., Deng, P., Li, X.-B., et al. (2017). AFF1 and AFF4 differentially regulate the osteogenic differentiation of human MSCs. Bone Res. 5:17044. doi: 10.1038/boneres. 2017.44
Zhu, Y., Pe'ery, T., Peng, J., Ramanathan, Y., Marshall, N., Marshall, T., et al. (1997). Transcription elongation factor P-TEFb is required for HIV-1 tat transactivation in vitro. Genes Dev. 11, 2622-2632. doi: 10.1101/gad.11.20.2622

Conflict of Interest: The authors declare that the research was conducted in the absence of any commercial or financial relationships that could be construed as a potential conflict of interest.

Publisher's Note: All claims expressed in this article are solely those of the authors and do not necessarily represent those of their affiliated organizations, or those of the publisher, the editors and the reviewers. Any product that may be evaluated in this article, or claim that may be made by its manufacturer, is not guaranteed or endorsed by the publisher.

Copyright (c) 2021 Chen, Qiang, Zhang, Cao, Wu, Jiang, Ai, Ma, Dong, Gao, Li, $\mathrm{Lu}$, Zhang and Chai. This is an open-access article distributed under the terms of the Creative Commons Attribution License (CC BY). The use, distribution or reproduction in other forums is permitted, provided the original author $(s)$ and the copyright owner(s) are credited and that the original publication in this journal is cited, in accordance with accepted academic practice. No use, distribution or reproduction is permitted which does not comply with these terms. 